\title{
Mobisense Testbed: Merging User Perception and Network Performance
}

\author{
[Invited Paper]
}

\author{
Pablo Vidales \\ Deutsche Telekom \\ Laboratories \\ Berlin, Germany \\ Pablo.Vidales@telekom.de \\ Frank Steuer \\ Technische Universität Berlin \\ Berlin, Germany \\ Frank.Steuer@dai-labor.de
}

\author{
Niklas Kirschnick \\ Deutsche Telekom \\ Laboratories \\ Berlin, Germany \\ Niklas.Kirschnik@telekom.de

Marcel Wältermann
Deutsche Telekom
Laboratories
Berlin, Germany
Marcel.Waeltermann@telekom de

\author{
Blazej Lewcio \\ Deutsche Telekom \\ Laboratories \\ Berlin, Germany \\ Blazej.Lewcio@telekom.de
Sebastian Möller
Deutsche Telekom
Laboratories
Berlin, Germany \\ Sebastian.Moeller@telekom.de
}

\begin{abstract}
The convergence of different commercial wireless technologies poses new opportunities for always-on connectivity and nearly ubiquitous access in Next Generation Networks (NGNs). The proposed integrated virtual access platform frees users from the fixed locations, and allows them to enjoy services while they are on the move. However, the possibility to seamlessly roam (on demand) across wireless technologies is a must in NGNs. Even though a wide body of research has been done on seamless mobility, a thorough analysis of user perception of this phenomenon is required in order to successfully design, and further improve, always-on services and mobility management solutions. This paper describes a testbed specially built to investigate the user perception of mobility in NGNs. The Mobisense testbed enables the mapping of user experience to network conditions, focusing on phenomena caused by a user roaming across diverse wireless technologies and the impact on applications and services. The Mobisense setting helps in the analysis of IP services, in particular experiments were done to study user perceived quality of Voice over IP in NGNs. This testbed allows (1) the migration of VoIP calls across heterogeneous wireless networks, (2) network application data tracking, (3) simulation of user mobility patterns, (4) and audio codec changeovers while roaming between networks.
\end{abstract}

\footnotetext{
*Pablo Vidales is member of the National System of Researchers (SNI) in Mexico since January 2007.
}

Permission to make digital or hard copies of all or part of this work for personal or classroom use is granted without fee provided that copies are not made or distributed for profit or commercial advantage and that copies bear this notice and the full citation on the first page. To copy otherwise, to republish, to post on servers or to redistribute to lists, requires prior specific permission and/or a fee.

TRIDENTCOM 2008, 17th- 20th Mar 2008, Innsbruck, Austria. Copyright @ 2011- 2012 ICST ISBN 978-963-9799-24-0 DOI 10.4108/icst.tridentcom.2008.3080

\section{INTRODUCTION}

The rapid growth of mobile computing and deployment of wireless networks drive the demand for IP services on the move - ending dependencies between services and physical locations. Internet services will be decoupled from the device's point of attachment to the network. Solutions such as Mobile IP [20], [29] and SIP [34] produce a feeling of freedom as users are able to roam among wireless technologies while performing their normal tasks (email, Internet browsing, etc). This service continuity demands support for handovers between networks, as the trade offs between coverage and bandwidth are still defined by the link characteristics of each wireless technology. Therefore, there is no unique technology that is able to offer ubiquitous coverage and fulfill all different applications' demands at the same time. A recent architecture for access networks proposes a conglomerate of wireless networks that intends to enable always-on access, and to provide nearly ubiquitous coverage (see Figure 1 for a time-line evolution of mobile computing). In this context, it is an open challenge to maintain the service quality and satisfy user expectations while roaming across such networking scenario. Moreover, voice quality assessment methods for conventional communication already exist [30], however, new phenomena resulting from mobility in these heterogeneous environments have not been investigated - or even understood.

The trade offs between network characteristics, when mobile users roam in NGNs, should be considered in order to meet user expectations - as perception is a subjective criterion. Most efforts have targeted the reduction of handover latency, signaling overhead, and packet losses, while neglecting the user perception of such networking phenomena. To our knowledge, a thorough assessment of user perception of mobility is Next Generation Networks has not been done before. A twofold approach (user and networking views) is still a missing piece in the puzzle towards seamless mobility. 


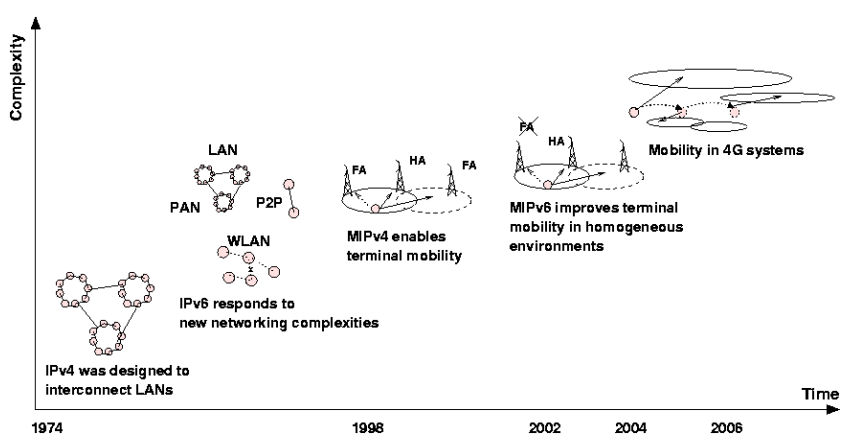

Figure 1: Evolution path towards seamless mobility and Next Generation Networks

This paper describes our ongoing efforts done to answer the following related questions:

- Which quality impairment arise from mobile users roaming across heterogeneous wireless technologies?

- Which are the potential benefits of performing heterogeneous (vertical) handovers in terms of user perception?

- Which are the potential advantages of codec adaptation/changeover in ongoing VoIP calls?

- How does a user perceive audio bandwidth transitions?

The Mobisense project connects the mobility phenomena and user perception in order to better understand the aforementioned issues. These knowledge is fundamental to comprehend how users will perceive mobility in NGNs and to provide better solutions for mobile applications. The Mobisense project aims at extending the current quality prediction models by introducing mobility events like network handover or codec changeover [38]. Mobility management and service adaptation solutions could be designed considering user expectations and not only the "opaque" data extracted from lower layers.

In this paper, we describe the design and implementation of an experimental setup used to investigate the user perception of VoIP calls in wireless heterogeneous networks. This testbed enables the analysis and assessment of various mobility phenomena from the networking and usability perspectives, and it allows the correlation between networking conditions and of user perception.

The rest of this paper is organized as follows. The next section includes the main concepts related to this work and it summarizes the most relevant related work. The Mobisense testbed is presented in Section 3 by listing the technical requirements, software and hardware components, and its main features. Section 4 describes a software solution that was developed, as part of the Mobisense testbed, to support audio codec changeovers. A summary of the results gathered from the first experiments using the testbed is included in Section 5. The paper ends with Section 6 that includes an outlook and conclusions.

\section{RELATED WORK}

Most of the existing work in this area deals with the design and enhancement of IP mobility management at the network plane, with solutions like MIP [20], [29], SIP [34], and HIP [27] that have even been standardized and embraced by the industry. In [37], the authors propose different improvements to the MIP solution, targeting issues such as reduction of handover latencies [11] or packet loss [9]. However, most previous papers overlook the impact of the network layer on the user perception; sometimes completely ignoring this point. The purpose of this paper is to trigger a deep debate on this topic. For this reason, the rest of the work is always discussed following a twofold inspection from the networking and perception angles.

On the networking viewpoint the following phenomenon is studied: terminal mobility and the impact of migrating the point of attachment between different access networks on user perception. In the Mobisense testbed, the support for transparent mobility of the Mobile Node (MN) is enabled by Mobile IP version 4 (MIPv4) [29]. The scope of this research includes a thorough assessment of horizontal (i.e., homogeneous) and vertical (i.e., heterogeneous) mobility [11]. In addition to the mobility aspects, the network conditions are altered during handovers in a controlled scenario, for example increasing network delay or packet loss. Another fact under scrutiny is speech codec changes during VoIP calls [22], mainly focusing on changes between wideband-narrowbandwideband. Finally, VoIP over WiFi in broadband sharing scenarios $[3,35]$ is also in scope of research.

From the user perception angle, the phenomena listed above is correlated with data extracted from listening and conversational subjective tests [8]. The listening tests are performed using prerecorded speech samples of VoIP calls under certain controlled conditions emulating the phenomena in focus. Then, the Mean Opinion Score (MOS) [17] reflecting user perception, is obtained from the subjective tests. In addition, the Mobisense testbed has been extended to allow carrying out conversational tests.

The overall experimental process is supported on various standard methodologies to measure user satisfaction over a complete VoIP call [8] and the recency effect on VoIP calls [14]. For the prediction of the user perception there are several methods available that can be divided into parametric models (e.g., the so-called E-Model [16]) and signal-based models. Signal-based models, in turn, can be classified in referenced models (e.g., PESQ [18]) and unreferenced models (e.g., [23]). The referenced models have the drawback that they need the original and the degraded speech sample to obtain the results. On the other hand, the unreferenced models do not describe the user perception as well as the referenced models [32]. There are many investigations that use these types of methods to evaluate quality of VoIP [30].

In sum, the global objective of this methodology is to analyze the networking phenomena as perceived directly by mobile users, and the use of the results in the decisionmaking processes associated to various mobility scenarios and changes in service levels. There are already a few seminal research papers in this direction, for example in [31] the authors introduce OverPhone, which is a VoIP overlay 
Table 1: Hardware and software on the Mobisense testbed

\begin{tabular}{|c|c|c|}
\hline Entity & Hardware & Software \\
\hline 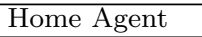 & Cisco 2620XM & $\overline{\text { IOS } 12.2(8 \mathrm{r})}$ \\
\hline Mobile Node & $\begin{array}{l}\text { IBM T60 } \\
\text { HSDPA Data card } \\
\text { Flarion Data card } \\
\text { IEEE } 802.11 \mathrm{a} / \mathrm{b} / \mathrm{g}\end{array}$ & $\begin{array}{l}\text { OpenSuse 10.2 } \\
\text { PJPROJECT 0.5.10.3 } \\
\text { SecGo Mobile IP } 3.2 \\
\text { NAT-traversal module } \\
\text { TCP server and client } \\
\text { UDP sender } \\
\text { tcpdump, netem }\end{array}$ \\
\hline $\begin{array}{l}\text { Correspondent } \\
\text { Node }\end{array}$ & IBM T60 & $\begin{array}{l}\text { OpenSuse } 10.2 \\
\text { PJPROJECT 0.5.10.3 } \\
\text { TCP client, tcpdump }\end{array}$ \\
\hline
\end{tabular}

network deployed on PlanetLab [13] for its evaluation. The OverPhone network uses the E-model to take routing decisions based on the user perception. The authors include results from several experiments performed on PlanetLab, concluding that the quality of VoIP calls can be increased by using knowledge from user perceived quality to derive routing decisions. The experiments showed that the amount of low quality talk-spurts was reduced by the path-diversity offered by the overlay network by $20 \%$. In contrast to the present work, the perception data used to make routing decisions was not based on subjective tests, as this is a wired environment and the E-model is a good fitting. However, to include new phenomena in the decision process, e.g., wireless heterogeneous mobility, performing subjective tests would be recommended to adjust existing perception models.

In [25], the E-model is used to verify the results of a hybrid adaptive-fixed playout algorithm that uses Network Time Protocol (NTP) [26] to extract delay measurements. Then, using these measurements the receiver creates a delay profile before setting a fixed playout delay. The algorithm was deployed on a testbed specially build for this purpose [24]. The E-model was used, once more, to evaluate the effect of the proposed algorithm. The results showed that the received $\mathrm{R}$-value from the E-model can be improved by 2 to 25 times by using the solution proposed by the authors.

The User Satisfaction Index (USI) is developed in [12] to quantify the VoIP user satisfaction. The model behind the USI is derived from the duration of Skype [4] calls and network conditions, e.g., bit rate, jitter, and RTT. Therefore, the models based on the USI do not require auditory tests, reducing the cost of the experimental work. This is an advantage compared to other ITU models, e.g., PESQ, as metrics are easier to obtain and calculate in real time. In [12], the USI is validated by an independent set of metrics for voice interactivity derived from user conversation patterns, and the impact of the bit rate, jitter, packet loss, and delays are evaluated to provide hints about the priority of these metrics.

Testbeds dealing with IP mobility have been deployed. A pioneer testbed in this area is the BIB3R testbed [36] that was designed to provide a mobile network operator testbed which emulates NGNs. The main design considerations were to be able to easily add new access technologies, support for IPv4 and IPv6, mobility support, mechanisms to provide Quality of Service (QoS), AAA and PSTN - IP integration. The
BIB3R testbed has been used by different research institutes for their work. Examples of these works are interference measurements of Bluetooth and IEEE 802.11 WLAN and experiments on a Mobile IPv4 based single sign on mechanism, Mobile IPv6 terminal mobility with All-IP application support, and Video on Demand with adaptive QoS. However, the BIB3R testbed has not been employed for user perception studies, nor for mobility perception in NGNs.

In [15], the implementation of a testbed to emulate a wireless Internet access provider is presented. The main features of this testbed are multimedia call setup using SIP signaling, seamless mobility features, fast handovers, and QoS features for mobile clients. Although the testbed was realized to use $\mathrm{IPv}_{4}$, it was extended for IPv6 in order to study handover delay using SIP over IPv6 [28]. However, perception aspects were not in the scope of this work.

\section{TESTBED}

The Mobisense testbed was explicitly deployed to assess the user perceived quality of mobility in NGNs using VoIP as the case application. In order to do this, the Mobisense testbed should support experiments involving: collection of speech samples, execution of network handovers and codec changeovers, gathering of network traces, and modification of network conditions. The following functional requirements can be derived from these processes:

- The mobile terminal should have connectivity to at least two different wireless access technologies like WiFi, Flash-OFDM, WiMAX, UMTS/HSDPA or GPRS/EDGE.

- The mobile terminal should be able to seamlessly migrate a connection between these access networks (like it can be done with Mobile IPv4 or SIP).

- Network traces should be collected during the voice calls to allow further evaluation of the network conditions and codec changeovers.

- There should be the possibility to degrade the network conditions artificially, i.e., to increase delay, jitter, packet loss, and additional traffic to the network connection in use.

- A possibility to use a broadband sharing environment (using IEEE 802.11 technology).

- A VoIP call has to be established between the mobile terminal and a fixed host with a stable Internet connection to measure only the impact of the wireless path on audio quality.

- The audio stream of the VoIP call must be recorded for further analysis and for the listening and conversational tests.

- The VoIP client should support wideband and narrowband speech codecs with different bandwidth requirements.

- The VoIP client must be able to switch between different speech codecs during an ongoing call.

- A jitter buffer monitoring to track the codec frames in the VoIP application should be implemented. 


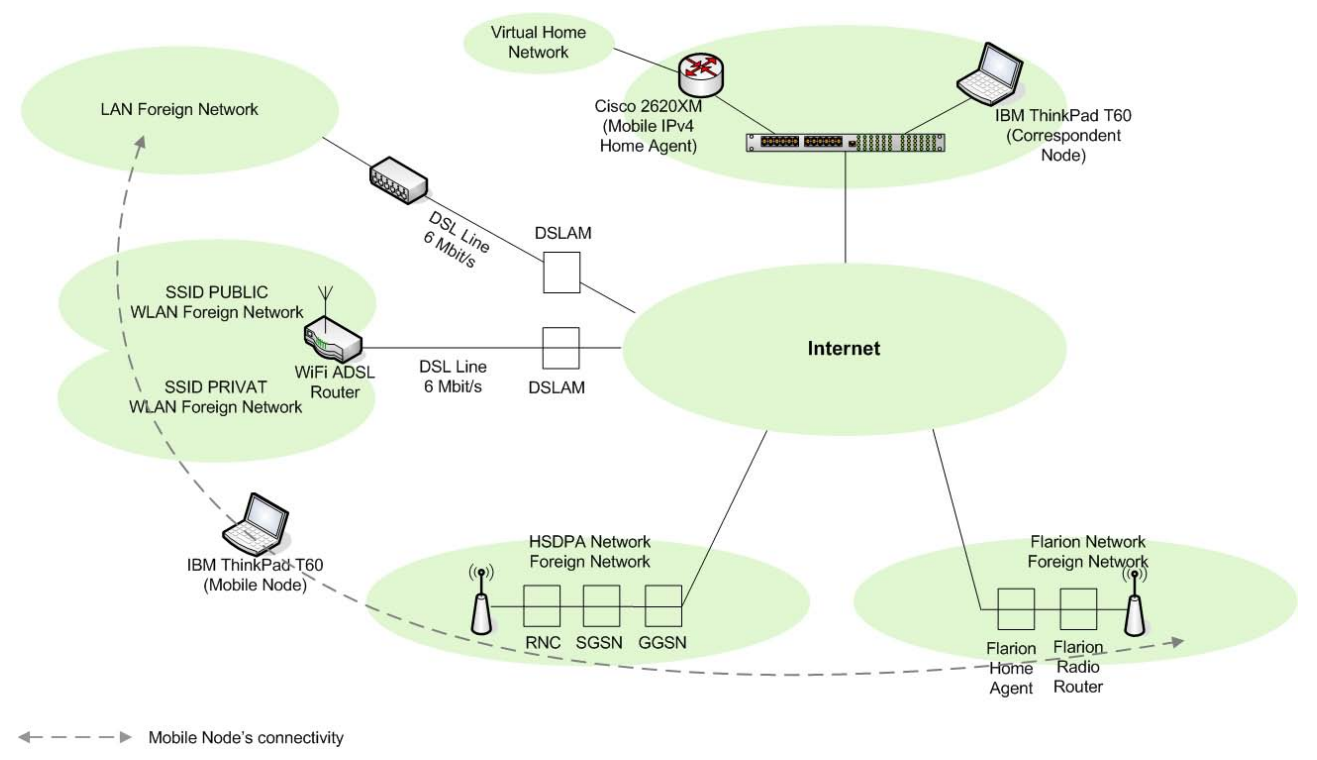

Figure 2: Network-centric view on the Mobisense testbed

These necessities had to be fulfilled for the success of this research. Some of them were realized using off-the-shelf hardware and software components. However, other parts were specially implemented for the Mobisense testbed. For example, the PJPROJECT client [10] was extensively modified to cope with the demanded features: codec changeover (Section 4) and jitter buffer monitoring.

\subsection{Hardware and software components}

The Mobisense testbed uses Mobile IPv4 as a solution to enable seamless handovers between different radio access technologies. Mobile IPv4 requires a Home Agent (HA), a Mobile Node (MN), and a Correspondent Node (CN) to build a working system. The access networks emulate an integrated NGN conformed by the following technologies: LAN, WiFi, Flash-OFDM, and UMTS/HSDPA. The CN and MN were deployed on laptops with Linux 2.6.18.2, and the HA is a Cisco 2620XM router with Cisco IOS 12.2(8r), supporting MIPv4 (see Table 1).

Mobile IPv4 was deployed as the mobility management protocol because all involved access networks support IPv4. As Mobile IP client, the SecGo implementation [2] was chosen because it provides a telnet interface for remote control [6] and supports NAT traversal [21]. As the VoIP framework, the PJPROJECT was selected and extensive modifications have been made to fulfill the testbed demands. The tcpdump [5] and wireshark [7] tools are used for trace collection and evaluation. netem [1] is used to enable changing the network characteristics in terms of adding delays, packet loss, packet duplication, packet corruption, and packet re-ordering. Finally, as shown in Figure 3, a TCP-based client/server application has been implemented to centralize the overall control of the test settings. In addition, an UDP sender has been implemented to enable the remote control of the VoIP client.

\subsection{Testbed deployment}

The Mobisense experimental setting described was specially deployed in order to investigate the relationship between mobility in NGNs and user perceived quality of VoIP services. Figure 2 shows the Mobisense testbed network architecture and its hardware components. The Mobisense testbed supports connectivity to six networks, as attachment points to the Internet, that are based on different (wireless) technologies: two LAN networks (one home and one foreign network), two foreign WLAN networks, one foreign UMTS/HSDPA network, and one foreign Flash-OFDM network. The home LAN network is directly connected to the Internet and is the fix attachment point for the Home Agent and Correspondent Node; the Mobile Node can roam between the five remaining networks. As shown in Figure 2 , the MN could connect to the foreign LAN and then it can roam to any of the two foreign WLAN networks, supplied by a DSL line as the backhaul. Moreover, the MN could log on to a public (foreign) UMTS/HSDPA network, as well as communicate with the Flash-OFDM RadioRouter that forwards packets to the Internet via the Flash-OFDM internal Home Agent. The Flash-OFDM access network is provided by the BIB3R testbed [36] and it is based on Flarion RadioRouter version 1.1 - a testing license in the UMTS frequency band has been granted by the German regulator enabling operational measurements.

Arising from the complex setting described in this section, two further requirements were posed to the design: (1) the $\mathrm{CN}$, acting as the counterpart of the VoIP call generated by the MN, has to be remotely controlled and (2) all software components should have an interface for remote management. Figure 3 depicts interconnections of software components deployed on the Mobile and Correspondent Nodes. The central point of the architecture is a controlling script on the MN that synchronizes the execution of all other software components. For example, the controlling script starts tcpdump to collect network traces, tc to adjust netem module, telnet to communicate with the Mobile IP client, UDP 
sender to control the PJPROJECT client, and the TCP client/server. The TCP client/server is employed twofold. At first, it runs the PJPROJECT client as a background process. Secondly, it controls the software installed on the Correspondent Node. Thus, the TCP client/server is also integrated to the CN, where it starts tcpdump and the PJPROJECT client, and controls the UDP sender.

\subsection{Functionality}

The Mobisense project aims to evaluate the effects of mobility on the perceived quality of real time services. Therefore, the testbed was designed to provide following features:

- Access to different network technologies

- Ability to change the network connection (network handover)

- Possibility to artificially change network conditions

- Record network traces

- Share available bandwidth using DSL and WLAN technologies

- Perform VoIP calls over the networks

- Support for different voice codecs (e.g. narrowband and wideband)

- Possibility to change voice codecs (codec changeover)

- Ability to record voice samples

- Ability to record internal parameters of the VoIP application

With these features there is much potential to evaluate different effects and their impact on the quality of VoIP calls:

- Different networks

- Different codecs

- Network handovers

- Bandwidth sharing

- Codec changeovers

- Network handovers in connection with codec changeovers

To evaluate the perceived quality, experiments with the above listed phenomena can be conducted. The resulting audio samples will be recorded and then used to run auditive tests with human listeners. During these listening tests, people are asked to rate the quality of different samples. These results can be used to rate different phenomena and as an input for the quality prediction model. Beyond this, the network-centric effects of the network handovers and codec changeovers can be evaluated by means of network trace recording ability of the testbed. Furthermore, through its open nature the testbed can be easily extended in different directions, which will be mentioned in Section 6 .

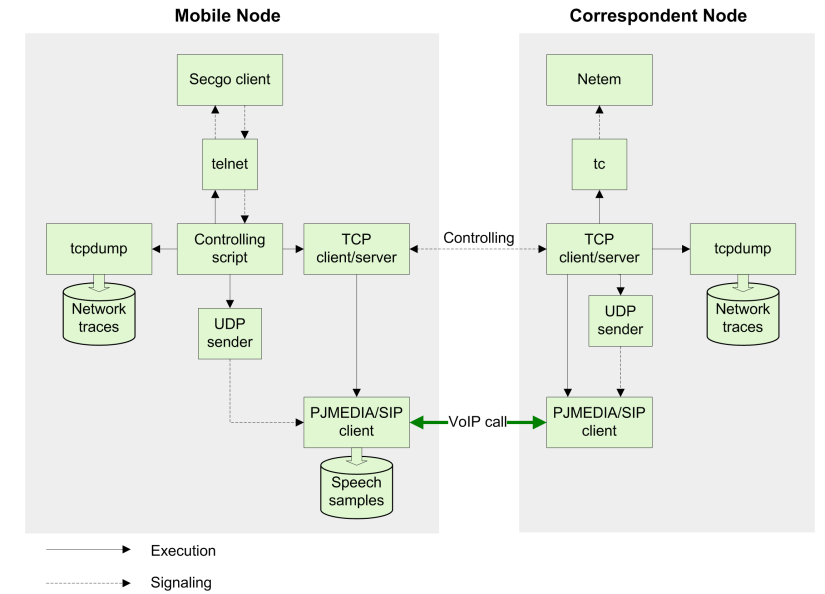

Figure 3: Intercommunication between testbed software components

\section{CODEC CHANGEOVER SOLUTIONS}

The present work on user perception of audio bandwidth fluctuations during ongoing VoIP calls uses codec changeover solutions that were developed as part of Mobisense testbed $[22,38]$. These solutions are based on the SIP/SDP parameter renegotiation [33], parallel media stream establishment, and RTP packet filtering. This enables the VoIP application to conceal potential packet loss due to codec changeovers, eliminating application related impacts on the audio stream quality.

\subsection{Media flow in PJPROJECT}

The codec changeover solutions implemented in the Mobisense project are based on the PJPROJECT 0.5.10.3 framework [10]. Figure 4 depicts the structure of this SIP client. The main interchange point of the audio signal is the Conference Bridge. This module is the interface between media streams and the Sound Device Port that, in turn, connects to the Sound Device API. Every Media Stream consists of a codec, a jitter buffer, and a RTP session control instance. The Media Transport module acts as an internal network interface; inherently, one Media Stream connects to one Media Transport unit, and one Media Stream build one Media Session.

\subsection{Requirements of a codec changeover solu- tion}

According to the the above described VoIP client architecture, the codec changeover design must cope with the following problems:

- The RTP session may reject packets with incorrect header information, what includes codec related changes of payload type information and abrupt changes of packet sequence number. Therefore, the media stack must be able to serve more then one RTP header type during the codec changeover process. Accordingly, the payload type identifier and the sequence number are the significant header fields. 


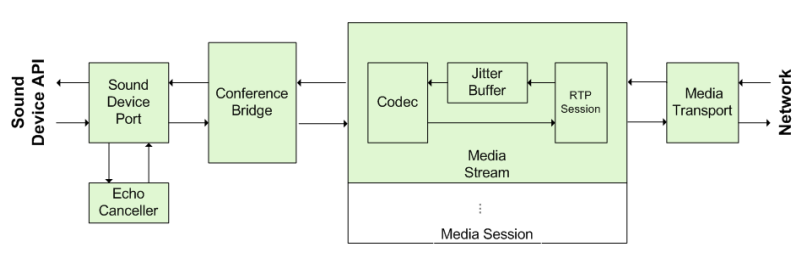

Figure 4: Media flow in PJPROJECT

- It must be assured that during codec changeover, represented by two simultaneously active media streams within one media session, only one of the streams sends data to the network interface. This measure avoids sending same data.

- The current jitter buffer must be played out and must not be destroyed immediately. Two benefits result from such a procedure: On the one hand, it prevents losing data in the old jitter buffer. On the other hand, the additional playout time allows to bridge the prebuffering time in the new jitter buffer.

\subsection{Codec changeover solutions}

Three codec changeover solutions were considered: Hard Codec Changeover (HCC), Soft Codec Changeover (SCC) with a separate Media Transport (MT), and SCC with a shared MT [22]. While the first one does not fulfill the stated requirements, it serves as a baseline solution to compare against the SCC approach, which eliminates application related audio stream interruptions. This paper describes only the HCC and SCC with a shared MT, and skips the description of the SCC with a separated MT unit, as its structure is similar to the prime SCC solution (for further information on the changeover solutions, see [38]). Both SCC solutions differ in the internal RTP packet routing (RTP filtering) required in the prime solution because of network socket sharing. The use of one network socket aims at preventing possible firewall problems related to dynamic RTP port changes during ongoing VoIP calls [33]. The three requirements of the codec changeover solution are fulfilled.

\subsubsection{Hard Codec Changeover}

This solution is a break-before-make method. In HCC, the Media Streams that serve the old and new codecs are substituted immediately without respecting the codec changeover requirements. Thus, there is no possibility to play out currently buffered data and to bridge the pre-buffering time in the new media stream. This, in turn, results as an applicationrelated gap in the audio stream.

\subsubsection{Soft Codec Changeover with a shared Media Transport}

The SCC solution realizes the make-before-break strategy. To serve both involved RTP payload types, SCC creates a new Media Stream before the old one is destroyed. Figure 5 depicts the media flow of this approach. Only the new Media Stream (MS) is connected to the Media Transport unit and is capable of network connectivity. Thus, the new RTP session instance needs to verify the RTP payload type of the incoming packets and forward them to the old MS, if necessary. Both of the Media Streams are bidirectional

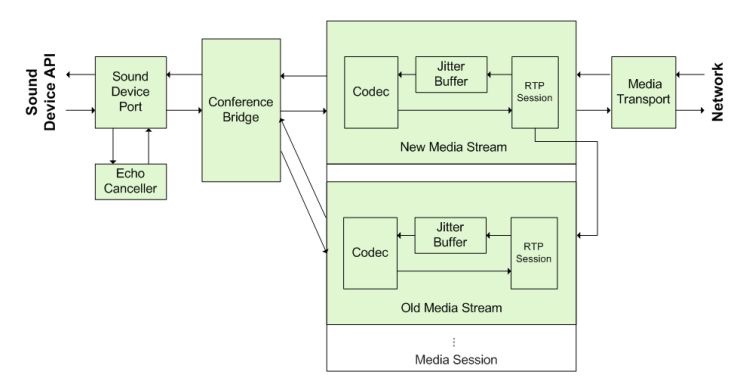

Figure 5: Soft Codec Changeover with a shared Media Transport

attached to the Conference Bridge. However, the old MS is not connected to the Media Transport and can only receive RTP packets forwarded from the new MS.

Table 2 summarizes the main features of the proposed codec changeover solutions. Both presented solutions prevent unpredictable behavior of firewall systems. Nevertheless, a trade-off between the codec transition quality and the implementation effort exists. The implementation effort increase with the desired codec changeover quality; for more design and performance details, see $[22,38]$.

For the experiments presented in the next section, the SCC with a shared MT was employed to prevent any application related impact on the quality of transmitted voice. Hence, this makes it possible to map user experiences to the network conditions and correctly evaluate the impact of mobility on user perception.

\section{INITIAL EXPERIMENTS}

The Mobisense testbed described in the foregoing sections allows to investigate the implications that changes in the network layer have on the application layer, and, ultimately, onto the perception of users roaming in NGNs. The impact on speech quality of VoIP is considered as a first case study here. During the initial experiments the Mobile Node was fixed and within the range of all the wireless networks. Therefore, a make-before-break policy was always possible when performing network handovers, resulting in optimal network conditions.

The impact of vertical handover latencies was significantly reduce by forcing the MN to connect to the destination network before the current one becomes unreachable, when performing an upward handover. In the opposite case, during a downward handover, the MN disconnects from the current network until the destination network is reachable.

Table 2: General comparison of codec changeover approaches

\begin{tabular}{|l||c|c|c|}
\hline & $\begin{array}{c}\text { Implementation } \\
\text { complexity }\end{array}$ & $\begin{array}{c}\text { Firewall } \\
\text { problems }\end{array}$ & $\begin{array}{c}\text { Transition } \\
\text { quality }\end{array}$ \\
\hline \hline HCC & low & no & low \\
\hline SCC (separated MT) & middle & possible & high \\
\hline SCC (shared MT) & high & no & high \\
\hline
\end{tabular}


The most critical aspects of mobility in NGNs are certainly vertical handovers along with the adaptation of the application layer to the newly encountered network: The round-trip time and the transmission bandwidth may change considerably and abruptly. For ongoing VoIP calls, this translates into jitter buffer adaptation or codec changeover, respectively, potentially provoking packet loss. This, in turn, leads to a degraded audio stream. Additionally, an inherent chance in speech quality may occur due to a change to different speech codec.

Thus, the following parameters are foreseen to have the most impact on the speech quality of a VoIP call in Next Generation Networks: packet loss, delay, codec (changeover).

Extensive auditory tests were conducted in the past in order to investigate the impact of packet loss and delay on speech quality perception (e.g. [30]). Although wideband speech transmission (audio bandwidth of $50-7000 \mathrm{~Hz}$ or beyond) is more and more studied in the related literature, narrowband speech quality (300-3400 $\mathrm{Hz}$ audio bandwidth) has been investigated in far more depth. Instrumental speech quality models, which are able to predict user judgments collected in auditory tests, are available for narrowband speech (e.g., [16], [18]) and to some extent also for wideband speech (e.g., [19]).

However, the impact of mid-call codec changeovers on quality perception that go along with (or are provoked by) vertical handovers in the networking layer have not yet been investigated. Strong effects are especially expected when codecs of different audio bandwidth are seamlessly interchanged (e.g., from G.722.2 (wideband) to G.711 (narrowband)). Furthermore, additional packet loss and changes in delay might arise in parallel and lead to further degradations.

To obtain a first insight into such effects, an initial listening only test was conducted. The participants were asked to listen to preprocessed speech samples and judge the overall speech quality. The processing of the samples was performed using the Mobisense testbed. Controlling scripts (see Section 3) were compiled in order to generate the following phenomena: handovers among various wireless access networks (mobility), codec changeovers, and a combination of mobility and codec variations.

The listening test was conducted following the guidelines given in ITU-T Recommendation P.800, which prescribes the listening room specifications and test methodologies. Without going into details, the analysis of the test results reveals, amongst other things, that:

\section{- Packet loss is the degrading quality most factor.}

- Switching from a wideband codec to a narrowband codec is worse than sticking to the wideband codec, independently of network conditions.

- If codec switching is performed along with a network handover, the latter is perceptively negligible.

- Handovers are not a strong degradation factor for VoIP quality in NGNs.
Furthermore, the obtained results lead to the assumption that the following rank order of importance exists for the elements provoking a speech quality decrease: 1) Packet loss, 2) change in audio bandwidth due to codec changeover, and 3) network changeover. If two effects occur simultaneously, however, the picture may change depending on the respective degree of degradation. Thus, aiming at a high speech quality in NGNs, an appropriate mobility management and VoIP solutions should incorporate such "trade-off rules".

However, experiments with further conditions are needed to derive stable conclusions from the above assumptions. In order to simulate realistic calls, for example, the "Call Quality" methodology [8] may be adapted to NGN calls. In such an experiment, participants are asked to listen to one or two minute long samples that represent a specific call scenario. An interactive call is mimicked by asking the participant questions about the content in-between short passages in each scenario. The whole call is judged afterwards with respect to the overall quality. This methodology has the advantage that "memory effects" are inherently taken into account: Degradations occurring toward the end of a call appear usually most annoying.

Going one step further, conversational experiments can be performed with the Mobisense testbed as well: A pair of two conversation partners interact via an established VoIP connection according to a pre-defined scenario [30]. Simultaneously, certain degradation patterns are applied on the network and application layer. In conversational tests, the overall delay can be taken into account as well. Such tests, although most expensive in terms of time, effort, and money, approximate a realistic call at most. Such experiments are currently carried out at Deutsche Telekom Laboratories. The results and implications for user perception based mobility management and the derivation of new and modified speech quality models will be published in the future.

\section{OUTLOOK AND CONCLUSIONS}

So far, the testbed has been used to record audio samples of VoIP calls with different mobility-related phenomena (network handover, codec changeover, link degradations, etc.) as the input for auditory tests. To get quality ratings during real user interaction the testbed can be extended to an online version, i.e. that two people make a VoIP call using the Mobisense setup. This implies that all the phenomena described in this paper should be controlled in real-time, as the calls take place.

Another extension could be to extend the testbed with commercial mobile devices. To this end, the mobile devices have to act as mobile nodes and support MIPv4 (some commercial mobile phones are already MIPv4 enabled). In addition, PJPROJECT with the corresponding codec changeover extension has to be ported to the selected devices.

Another plausible extension could be to evaluate the quality of voice calls and mobility in NGNs not only for VoIP, but also for real time streaming video (e.g., mobile TV protocols). For this purpose, a video streaming client has to be installed on the Mobile Node and a video streaming server on the Correspondent Node, and the consequent modifications and extensions have to be realized. The client/server 
suite should support video codec changeovers to adapt to existing network conditions. All of these extensions could be combined to evaluate the effects of mobility events on the perceived quality of mobile video streaming and video conferencing.

The Mobisense project aims at evaluation of user perception in wireless networks and the impact of mobility on it. Therefore, a testbed that meets the requirements detailed in Section 3.2 was implemented. The main requirements were to provide the needed real-time service, the possibility to connect to several access networks, and the support for seamless handovers between these access networks during calls. Based on these requirements the decision was taken to use Mobile IPv4 as the mobility solution and an open source VoIP client that could be adapted to further needs. The following characteristic make the Mobisense testbed a good tool to evaluate user perception of mobility in NGNs:

- It is mainly based on open source software.

- It uses standard hardware components as well as software components.

- Due to this it is easy to extend to further needs.

The Mobisense testbed opens the possibility to use a realistic environment for real-time services in NGNs and to evaluate the quality of these services from the users' angle.

\section{Acknowledgments}

This study was partly supported by the Deutsche Forschungsgemeinschaft (DFG), grant MO1038/5-2.

\section{REFERENCES}

[1] Net:Netem - The Linux Foundation. http://www.linux-foundation.org/en/Net:Netem.

[2] Secgo Mobile IP Toolkit. http://www.secgo.com/.

[3] Website of FON. http://www.fon.com/.

[4] Website of Skype. http://www.skype.com/.

[5] Website of TCPDUMP. http://www.tcpdump.org/.

[6] Website of Telnet. http://www.telnet.org/.

[7] Website of Wireshark. http://www.wireshark.org/.

[8] Speech Processing, Transmission and Quality Aspects (STQ); Estimating Speech Quality per Call. ETSI TR 102506 v.1.1.1, Oct. 2006.

[9] N. Akkari, S. Tohme, and M. Doughan. Applying Anticipated Vertical Handover (AVHO) in Next Generation Networks. In Proceeding of Fourth European Conference on Universal Multiservice Networks (ECUMN 2007), pages 311-319. IEEE Computer Society, Feb. 2007.

[10] B.Prijono. Open Source SIP Stack and Media Stack for Presence, Instant Messaging, and Multimedia Communication. http://pjsip.org/.

[11] R. Chakravorty, P. Vidales, K. Subramanian, I. Pratt, and J. Crowcroft. Performance Issues with Vertical Handovers - Experiences from GPRS Cellular and WLAN Hot-Spots Integration. In Proceedings of the
Second IEEE International Conference on Pervasive Computing and Communications (PerCom'04), pages 155-164, Washington, DC, USA, Mar. 2004. IEEE Computer Society.

[12] K. Chen, C. Huang, P. Huang, and C. Lei. Quantifying Skype User Satisfaction. In Proceedings of the Conference on Applications, Technologies, Architectures, and Protocols for Computer Communications(ACM SIGCOMM 2006), pages 399-410. ACM Press, Sept. 2006.

[13] B. Chun, D. Culler, T. Roscoe, A. Bavier, L. Peterson, M. Wawrzoniak, and M. Bowman. PlanetLab: an Overlay Testbed for Broad-Coverage Services. ACM SIGCOMM Computer Communication Review, 33(3):3-12, July 2003.

[14] A. Clark. Modeling the Effects of Burst Packet Loss and Recency on Subjective Voice Quality. In Proceedings of Internet Telephony Workshop, New York, Apr. 2001.

[15] A. Dutta, J. Chen, S. Das, M. Elaoud, D. Famolari, S. Madhani, S. McAuley, M. Tauil, S. Baba, T. Maeda, N. Nakajima, Y. Ohba, and H. Schulzrinne. Implementing a Testbed for Mobile Multimedia. In Proceedings of the IEEE Global Telecommunications Conference 2001 (GLOBECOM 2001), volume 3, pages 1944-1949. IEEE Press, Nov. 2001.

[16] ITU-T Rec. G.107. The E-model, a Computational Model for Use in Transmission Planning. International Telecommunication Union, Mar. 2005.

[17] ITU-T Rec. P.800. Methods of Subjective Determination of Transmission Quality. International Telecommunication Union, Aug. 1996.

[18] ITU-T Rec. P.862. Perceptual Evaluation of Speech Quality(PESQ): An Objective Method for End-to-End Speech Quality Assessment of Narrow-Band Telephone Networks and Speech Codecs. International Telecommunication Union, Feb. 2001.

[19] ITU-T Rec. P.862.2. Wideband Extension to Recommendation P.862 for the Assessment of Wideband Telephone Networks and Speech Codecs. International Telecommunication Union, Nov. 2005.

[20] D. Johnson, C. Perkins, and J. Arkko. Mobility Support in IPv6. RFC 3775 (Proposed Standard), June 2004.

[21] H. Levkowetz and S. Vaarala. Mobile IP Traversal of Network Address Translation (NAT) Devices. RFC 3519 (Proposed Standard), May 2003.

[22] B. Lewcio. Assessment and Adaptation of Voice Quality in All-IP Heterogeneous Networks. Diploma thesis (unpublished), Aug. 2007.

[23] L. Malfait, J. Berger, and M. Kastner. P.563 - The ITU-T Standard for Single-Ended Speech Quality Assessment. IEEE Transactions on Audio, Speech 86 Language Processing, 14(6):1924-1934, Nov. 2006.

[24] H. Melvin and L. Murphy. Time Synchronization for VoIP Quality of Service. IEEE Internet Computing, 6(3):57-63, May 2002.

[25] H. Melvin and L. Murphy. An Evaluation of the Potential of Synchronized Time to Improve Voice over IP Quality. In Proceedings of IEEE International Conference on Communications 2003 (ICC 2003), volume 3, pages 1922-1926, May 2003. 
[26] D. Mills. Network Time Protocol (Version 3) Specification, Implementation and Analysis. RFC 1305 (Draft Standard), Mar. 1992.

[27] R. Moskowitz and P. Nikander. Host Identity Protocol (HIP) Architecture. RFC 4423, May 2006.

[28] N. Nakajima, A. Dutta, S. Das, and H. Schulzrinne. Handoff Delay Analysis and Measurement for SIP Based Mobility in IPv6. In Proceedings of IEEE International Conference on Communications 2003 (ICC 2003), volume 2, pages 1085-1089, May 2003.

[29] C. Perkins. IP Mobility Support for IPv4. RFC 3344 (Proposed Standard), Aug. 2002. Updated RFC 4721.

[30] A. Raake. Speech Quality of VoIP: Assessment and Prediction. John Wiley \& Sons, Sept. 2006.

[31] R. K. Rajendran, S. Ganguly, R. Izmailov, and D. Rubenstein. Performance Optimization of VoIP using an Overlay Network. Technical report, NEC America, Sept. 2005.

[32] A. W. Rix, J. G. Beerends, D. Kim, P. Kroon, and O. Ghitza. Objective Assessment of Speech and Audio Quality - Technology and Applications. IEEE Transactions on Audio, Speech $\&$ Language Processing, 14(6):1890-1901, Nov. 2006.

[33] J. Rosenberg and H. Schulzrinne. An Offer/Answer Model with Session Description Protocol (SDP). RFC 3264 (Proposed Standard), June 2002.
[34] J. Rosenberg, H. Schulzrinne, G. Camarillo, A. Johnston, J. Peterson, R. Sparks, M. Handley, and E. Schooler. SIP: Session Initiation Protocol. RFC 3261 (Proposed Standard), June 2002. Updated by RFCs 3265, 3853, 4320, 4916.

[35] M. Solarski, P. Vidales, O. Schneider, P. Zerfos, and J. Singh. An Experimental Evaluation of Urban Networking Using IEEE 802.11 Technology. In Proceedings of the 1st IEEE Workshop on Operator-Assisted(Wireless Mesh) Community Networks (OpComm 2006), Sept. 2006.

[36] F. Steuer, M. Elkotob, S. Albayrak, and A. Steinbach. Testbed for Mobile Network Operator Scenarios. In Proceedings of 2nd International Conference on Testbeds 8 Research Infrastructures for the DEvelopment of NeTworks \& COMmunities (TRIDENTCOM 2006). IEEE Press, Mar. 2006.

[37] P. Vidales, C. Bernardos, I. Soto, D. Cottingham, J. Baliosian, and J. Crowcroft. MIPv6 Experimental Evaluation Using Overlay Networks. Computer Networks, 51(10):2892-2915, July 2007.

[38] M. Wältermann, B. Lewcio, P. Vidales, and S. Möller. A Technique for Seamless VoIP-Codec Switching in Next Generation Networks. (Accepted in IEEE ICC 2008). 